\title{
The Latest in Telemetry Data Visualization Solutions
}

\author{
David N. Kortick \\ Product Development Manager \\ AstroNova Inc. \\ 600 East Greenwich Ave. \\ West Warwick, RI 02893 USA
}

\begin{abstract}
Summary
The importance of visualizing time-critical data in aerospace telemetry applications cannot be overstated. The latest data visualization solutions offer the ability for the telemetry facility to utilize strip-chart display software, real-time chart printers or combination systems for range safety, flutter testing, quick-look and other mission critical applications. These visualization and recording systems are designed to support both existing and new ground stations by offering analog as well as digital (network) interfaces. Sharing a common user experience (UX) design, today's telemetry data visualization solutions provide the immediate data display required by flight test engineers, range safety personnel and others. Advances in recording, display, data acquisition and security technologies are all part of the architecture of these recording systems, which are easily adapted to the needs of each user.
\end{abstract}

\section{Keywords}

visual display, data recording, recorder, chart recorder

\section{Topics}

Data Acquisition \& Sensors, Recording Technology 


\section{Introduction}

Data visualization has been a crucial component of telemetry systems for many decades. From early strip chart recorders with their unique ability to graphically represent continuous realtime data to the latest in both software and hardware-based recording systems, data visualization is used in many applications including flight testing, rocket and missile launch monitoring, range safety and satellite testing. The modern telemetry recording systems have constantly evolved by utilizing the latest technologies to fulfill the needs of the users.

\section{A Brief History of Telemetry Recording Systems}

Telemetry data was originally recorded on strip chart recorders. These systems were originally galvanometer-based pen-writing instruments. The typical recorder drew eight waveforms on eight separate pre-printed grids of $40 \mathrm{~mm}$ width. The point-of-writing mechanism changed over many years, from free-flowing capillary ink styli to pressurized ink, to heated elements on thermally sensitive paper. While these recorders served the telemetry community very well, the drawbacks to these methods include the mechanical limitations of the galvanometer for high frequencies, the high maintenance costs of these systems, the untidiness of ink-based recorders and the lack of communication for command and control.

Telemetry recorder technology evolved to include a light-beam oscillograph which marked a photo-sensitive paper. While this offered higher bandwidth signal recording, the photosensitive paper was expensive and did not archive well. The next technology evolution was the electrostatic array recorder which was the first introduction of digital recording in a previously analog field and offered advancements such as overlapping channels, alphanumeric annotation, grids, graphics, and host control capabilities. One of the shortcomings of the electrostatic array recorder was a digitization effect due to a recording technique that limited the output resolution.

The thermal array recorder followed shortly after the electrostatic array recorder. This printing method, almost exclusively the only type still used for telemetry today, has many benefits including high resolution (300 dpi) printing, high frequency response, flexible chart formats, and alphanumeric annotation. Full host control was a major advancement for thermal array recorders, finally closing the loop between telemetry processor and recording system. Over the many years since the introduction of the first thermal array recorder, features such as video display, large memory capacities and digital signal processing were added to these instruments. High-speed digital data inputs became widely used with the thermal array recorders, eliminating the high costs and constant need for calibration of digital-to-analog converters (DAC) at telemetry installations. 


\section{Telemetry Recording Today}

The data visualization provided by the telemetry recorder is still very relevant today. The instruments have become much more than basic print recorders and have now advanced into true multimedia recording systems. The additions of the large touch-panel display, intuitive graphical user interfaces (GUIs), large capacity storage media and networking are capabilities found in the modern telemetry recorders at many telemetry facilities. The latest of these systems employ standard data protocols and include advancements in real-time data visualization, recording and networking.

The trend with most telemetry facilities today is toward software-based solutions for the realtime visualization of the telemetry data. However, the ability to visualize the telemetry data in a format that is useful to the engineer also calls for a hardcopy output for many applications. What is important is that the user experience (UX) is the same for these visualization systems. As paper is no longer the primary storage medium, the telemetry recorder plays a smaller, yet no less useful role. Continuous paper output still offers many unique benefits to the user in applications such as quick-look and range safety. The physical paper recording is a reliable and tangible medium that can be quickly viewed, marked-up, and "flipped through" to see important data or trending patterns. All of the data needed to make a determination is located on the paper. This includes the waveform data itself, timing marks, IRIG timecode, text annotation, event marks and even highlights of areas deemed important by the engineer. While the strip chart printout itself can be an important part of the record, there are many technologies utilized in the recorder that augment this fundamental capability.

\section{Waveform Display and Video}

The most recent designs of the telemetry recorder utilize a high-resolution touchpanel display that gives the user the ability to visualize real-time waveforms, events, grids and timing information in a traditional telemetry waterfall format. These displays can be totally customized in terms of waveforms sizes and placement, colors, grid definition, timing marks and annotation. In addition to the real-time waveforms, other types of graphs such as XY plots and meter displays can be selected by the user. Figure 1 shows a typical display formatted for 8 channels.

These displays also offer an intuitive and familiar GUIs for local command and control of the recording system. Control panels can be standardized or customized by the facility for specific tests and missions. The control panel buttons seen at the top of Figure 1 are a good example. 


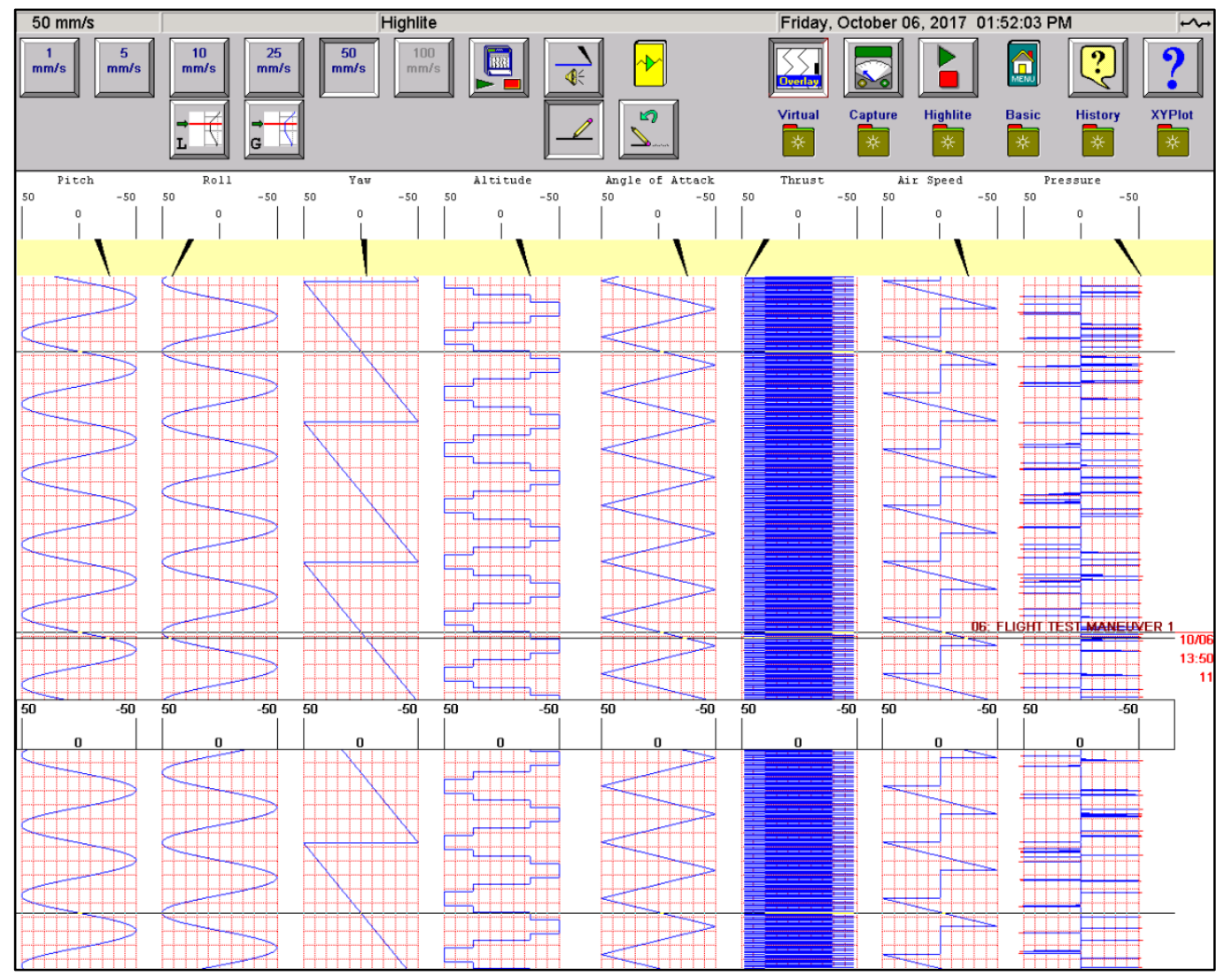

Figure 1: Screenshot of the recorder display

Another benefit of these instruments is the ability to have a video output available to allow the display to be sent to another monitor, auxiliary feed to a control room, or to a video mixing system. The latest designs utilize the High Definition Multimedia Interface (HDMI) for video output, due to the industry acceptance and standardization of this interface.

\section{Data Acquisition and Storage}

One of the most important aspects of the next-generation telemetry recorder is the ability to offer both digital and paper storage mediums, giving the engineer or analyst both options. In fact, the data acquisition capabilities of these systems have been greatly improved based on the requirements of the telemetry community. Sample rates of up to $200 \mathrm{kHz}$ per channel are now standard for installations where analog signal inputs are required. Enterprise-grade solidstate drives (SSD), with their consistent performance and reliability, have replaced magnetic hard drives in most of the newest installations. These drives are typically 3D NAND TLC flash memory, offering a good balance between performance and cost.

A typical use case for the data acquisition capability is for an application that requires an entire mission to be recorded locally on solid-state removable media, but the engineer or analyst may 
want to have a hardcopy printout of a few important areas of interest during the mission. The modern telemetry strip chart recorder makes this possible and offers maximum flexibility.

\section{Networking}

The ability to network multiple telemetry recorders is a requirement for almost all telemetry facilities. Many of these have central locations where one engineer or system operator can control the various recorders that are used in the facility. The newest systems offer Gigabit Ethernet (1000BASE-T) ports for interfacing to the equipment. This connectivity provides command and control, file transfer capability, web interfaces, digital data interfaces and even peer-to-peer communication. Figure 2 shows a typical block diagram for this interface.

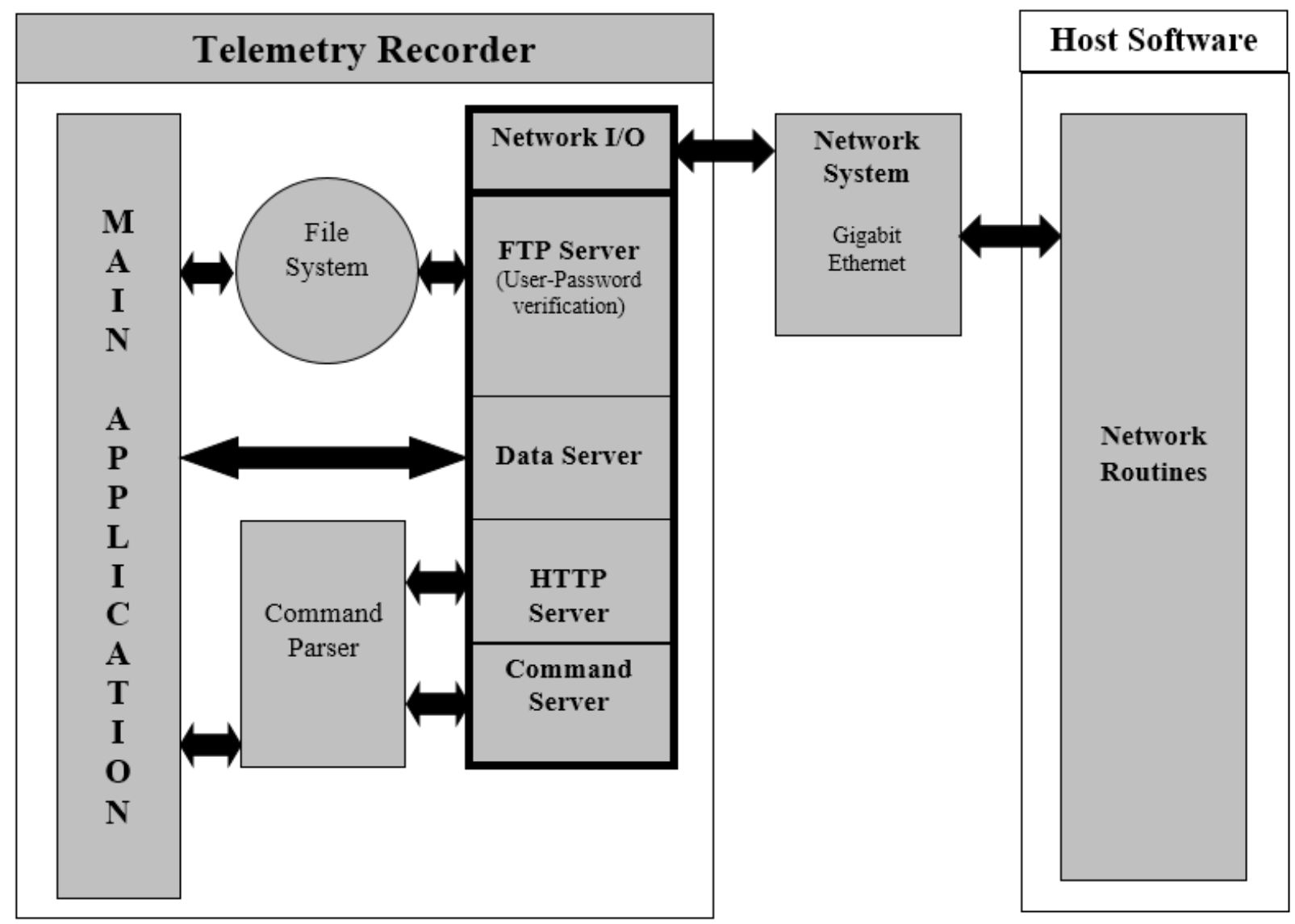

Figure 2: Block diagram of the network interfaces for the telemetry strip chart recorder

Modern telemetry recorders utilize both TCP and UPD protocols, depending on the need of the specific telemetry facility. FTP and HTTP protocols are also used in some facilities for file transfer and support of web-based client software. 
The digital data interface over Ethernet is one of the most commonly used capabilities of these recording systems, eliminating the need for DACs to provide analog data to the recorder. A higher-level data protocol is used to transmit data to the recorder. An example is an Ethernet digital data protocol which is optimized for strip chart recorder data with the use of the $\mathrm{min} / \mathrm{max}$ method and requires the host to send line segments based on the minimum and maximum waveform values for a slice of time, or $\Delta T$. The value of $\Delta T$ depends on the chart speed of the recorder. The data packets containing the line segments also include timing marks, IRIG time stamps and grid information that provide the host with advanced control over the visualization and printing of the data. Other benefits of a digital data interface include the reduced bandwidth requirements on the network and the advantage of glitch capture.

With glitch capture, the primary sample rate at the host can be much higher than the $\mathrm{min} / \mathrm{max}$ rate at which the packets are sent to the strip chart recorder. Therefore, a high-speed event can be seen as a minimum or maximum even at the relatively low rate that data is being sent across the network. In Figure 3, a waveform spike with frequencies of $1,10,100$, and $1000 \mathrm{~Hz}$ is shown with the full amplitude information as recorded.

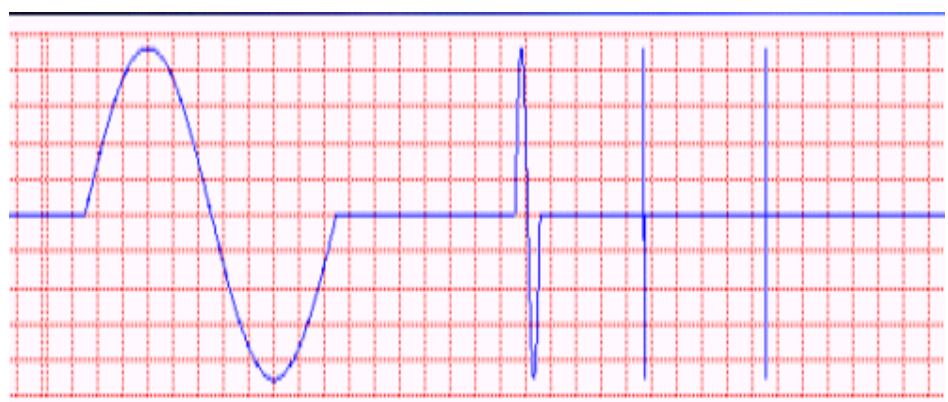

Figure 3: A sinusoidal waveform with glitch capture

\section{Security}

Security is an important consideration for any telemetry equipment today due to the proliferation of both physical and cyber threats. Malicious code, viruses and state-of-the-art intrusion methods are all concerns for the telemetry community. The next-generation telemetry recorders play a role in mitigating these threats by the use of the advanced technologies and operating systems in their designs.

From a physical standpoint, the recording system utilizes lockable and removable drive bays for the system and data acquisition media. This security is not only utilized for protection against malicious intent, but also to have the ability to use different media for distinct classified projects. 
The Windows 10 operating system is now used for the display and GUI functions of the recorder. This operating system, accepted by the DoD, offers the ability for Information Assurance (IA) compliance with the configuration standards of the Security Technical Implementation Guide (STIG).

\section{Technology-Based Data Visualization Solutions}

One of the challenges of telemetry facilities that use strip chart recorders is that many of these instruments are aging and utilize obsolete technology. These systems are used in a mission critical environment where failure is costly. Perhaps the most noteworthy development is the interest of many telemetry facilities in replacing these older systems with a COTS solution of customizable data visualization and printing methods that offer the same UX. This architecture is viable because of the ability to separate the waveform display capability from the printing capability in the latest recorder designs. Furthermore, the digital data protocol allows for a seamless integration between the source of the data and the endpoints for visualization or recording of the time-critical data. These endpoints are data visualization software stations, continuous network printers and strip chart recorders.

Figure 4 shows an example configuration where a telemetry processor is sending packets via the digital data protocol over Ethernet to these solutions. If only data visualization and local digital storage is required, then the visual display software station is utilized. If only strip chart recording is needed, then the continuous network printer can be used. If a full visualization, data recording and strip chart solution is required then the telemetry strip chart recorder is used. All three solutions share a common command and control structure as well as support the digital data protocol. 


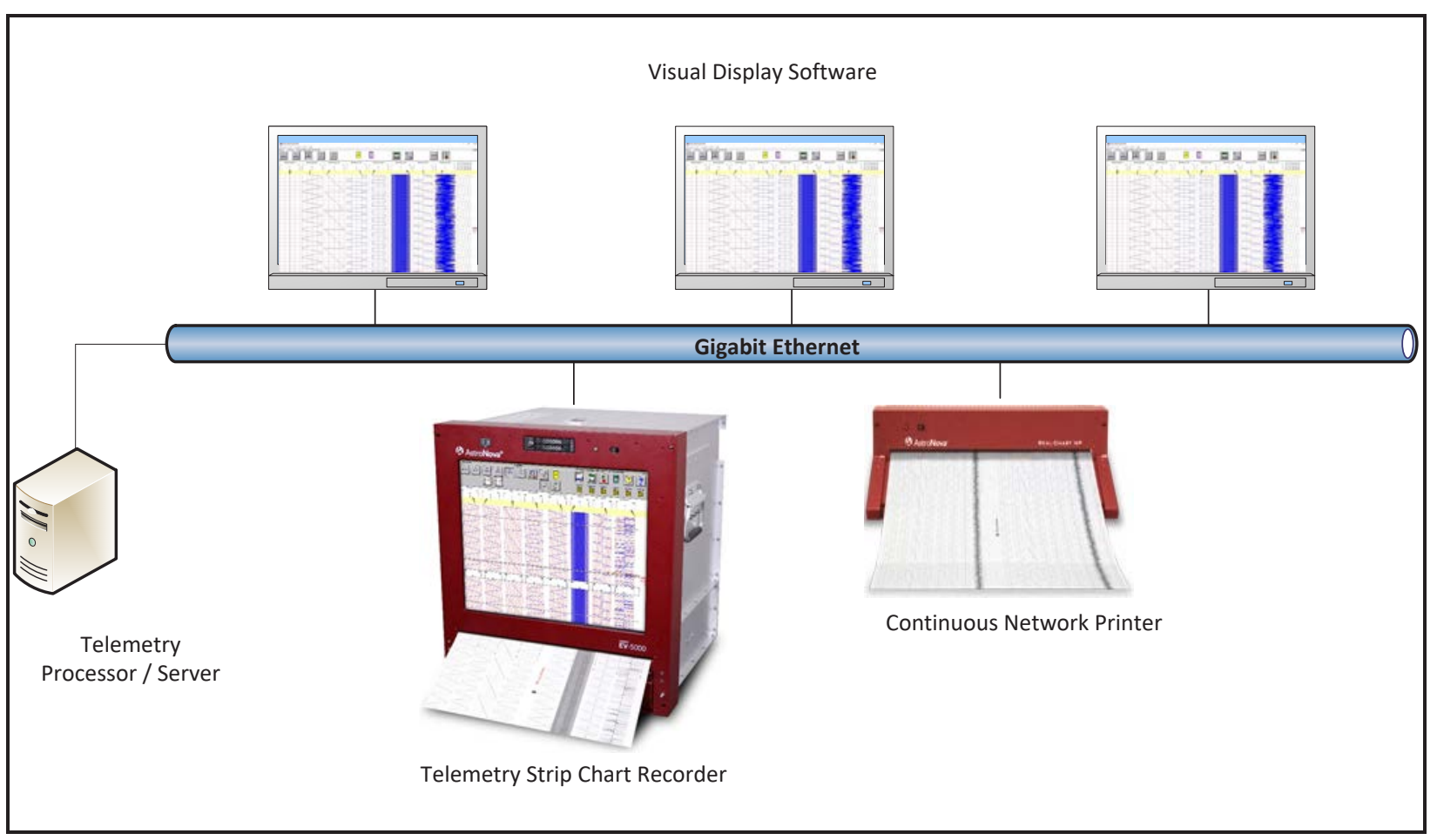

Figure 4: An example of a custom data visualization and recording configuration

The benefit to this architecture is that the telemetry facility can adapt to the needs of their customers. This flexible configuration can support different missions requiring different numbers of recorded parameters. An example is having many engineers or analysts viewing the data in real-time using the visual display software and having the ability to send data to the continuous network printer upon demand. Another example could be the addition of IRIG 106 Chapter 10 recording and playback capability.

\section{Conclusion}

The telemetry recording system is still a useful and viable part of the instrumentation for telemetry facilities throughout the world. While the need for a continuous printed record of telemetry data has been reduced due to other recording media, the requirements for some applications still call for the printed record. Today's next-generation systems not only fulfill that requirement but also offer the users and facilities much greater flexibility and capability in terms of data visualization and data acquisition. By offering a combination of software-based and hardware-based solutions, these telemetry recording systems are flexible in their configuration and can be adapted to any telemetry facility. The latest technologies and protocols used in today's designs also ensure that these systems meet current and future mission requirements. 